\title{
Backward Uniqueness for Parabolic Equations
}

\author{
L. EsCAURIAZA, G. SEREGin \& V. ŠVERÁK
}

Communicated by the Editors

\begin{abstract}
It is shown that a function $u$ satisfying $\left|\partial_{t}+\Delta u\right| \leqq M(|u|+|\nabla u|),|u(x, t)| \leqq$ $M e^{M|x|^{2}}$ in $\left(\mathbb{R}^{n} \backslash B_{R}\right) \times[0, T]$ and $u(x, 0)=0$ for $x \in \mathbb{R}^{n} \backslash B_{R}$ must vanish identically in $\mathbb{R}^{n} \backslash B_{R} \times[0, T]$.
\end{abstract}

\section{Introduction}

In this paper we prove backward uniqueness for solutions of

$$
\left|\partial_{t} u+\Delta u\right| \leqq M(|u|+|\nabla u|)
$$

in $Q_{R, T}=\mathbb{R}^{n} \backslash B_{R} \times[0, T]$, where $B_{R}=\left\{x \in \mathbb{R}^{n}:|x| \leqq R\right\}$. Our main result, Theorem 1 below, says that under natural regularity assumptions on $u$, any solution of (1.1) with controlled growth at infinity which vanishes at $t=0$ must vanish identically. The required growth condition is

$$
|u(x, t)| \leqq M e^{M|x|^{2}} .
$$

The main point of the theorem is that the values of $u$ at the parabolic boundary of $Q_{R, T}$ are not controlled by the assumptions. When $Q_{R, T}$ is replaced by $\mathbb{R}^{n} \times[0, T]$ the statement of Theorem 1 follows, for example from [2] and [17]. Papers [14, $18,20]$ also contain important related results. We remark that classical examples of A. N. Tikhonov show that Theorem 1 fails, even in $\mathbb{R}^{n} \times[0, T]$, when the growth condition (1.2) is slightly weakened.

One interesting consequence of Theorem 1 is that it settles a well-known problem in the regularity theory of the Navier-Stokes equations. This is explained in [19], where Theorem 1 was conjectured and proved for bounded $u$ in the simple 
case when (1.1) is satisfied with $M=0$. To formulate the result implied by Theorem 1 and [19], let us consider the classical Cauchy problem for the incompressible Navier-Stokes equations in $\mathbb{R}^{3} \times(0, T)$,

$$
\begin{aligned}
v_{t}+v \cdot \nabla v+\nabla p & =\Delta v \text { in } \mathbb{R}^{3} \times(0, T), \\
\operatorname{div} v & =0 \text { in } \mathbb{R}^{3} \times(0, T), \\
v(x, 0) & =v_{0}(x) \quad \text { in } \mathbb{R}^{3} .
\end{aligned}
$$

We assume that $v_{0}$ is a smooth divergence-free vector field with suitable decay at $\infty$. It is known that the problem (1.3) has at least one Leray-Hopf weak solution. (See, for example, [12, 7].) As proved in [19], Theorem 1 implies the following result.

Theorem. In the notation introduced above, assume that a Leray-Hopf weak solution $v$ of (1.3) is bounded in the space $L^{\infty}\left(0, T ; L^{3}\left(\mathbb{R}^{3}\right)\right)$. Then $v$ is smooth in $\mathbb{R}^{3} \times[0, T)$.

In fact, it can easily be seen from this and the local well-posedness of (1.3) in $L^{3}$ (see [11]) that the following slightly stronger statement is true: If $v_{0}$ is as above and a Leray-Hopf solution $v$ of $(1.3)$ is bounded in $L^{\infty}\left(0, t_{1} ; L^{3}\left(\mathbb{R}^{3}\right)\right)$ for some $t_{1} \leqq T$, then $v$ is smooth in $\mathbb{R}^{3} \times\left[0, t_{1}\right]$.

Theorem 1 is also of interest in control theory. MiCU \& ZUAZUA have shown in [16] the lack of null controllability of the heat equation on the half space for any positive time with $L^{2}$ control on the lateral boundary and for a large class of initial data. Theorem 1 shows that the same holds for operators $\partial_{t}+\Delta+b \cdot \nabla+c$ when $b$ and $c$ are bounded functions, and for domains containing the complement of a ball in $\mathbb{R}^{n}$. In fact, $b$ and $c$ can be considered as additional controls and the theorem says that under the growth assumption (1.2) null controllability by bounded controls for $u_{t}+\Delta u+b \cdot \nabla u+c u=0$ is not possible, except for the trivial case when $u$ vanishes identically. For exact controllability of the heat equation for bounded domains we refer the reader to $[10,15]$, for example.

The proof of Theorem 1 uses the following Carleman inequalities:

1. Set $\sigma(t)=t e^{-t / 3}$ and $\sigma_{a}(t)=\sigma(t+a)$. Then, there is a constant $N=N(n)$ such that the inequalities

$$
\begin{aligned}
& \left\|\sigma_{a}^{-\alpha-1 / 2} e^{-|x-y|^{2} / 8(t+a)} u\right\|_{L^{2}\left(\mathbb{R}^{n} \times(0,1)\right)} \\
& \quad+\left\|\sigma_{a}^{-\alpha} e^{-|x-y|^{2} / 8(t+a)} \nabla u\right\|_{L^{2}\left(\mathbb{R}^{n} \times(0,1)\right)} \\
& \leqq N\left\|\sigma_{a}^{-\alpha} e^{-|x-y|^{2} / 8(t+a)}\left(\Delta u+\partial_{t} u\right)\right\|_{L^{2}\left(\mathbb{R}^{n} \times(0,1)\right)}
\end{aligned}
$$

hold for all $\alpha \geqq 0, y \in \mathbb{R}^{n}, 0<a<1$ and $u \in C_{0}^{\infty}\left(\mathbb{R}^{n} \times[0,1)\right)$ verifying $u(., 0) \equiv 0$.

2. There is a constant $\alpha_{0}=\alpha(R, n)$ such that the inequalities

$$
\begin{aligned}
&\left\|e^{\alpha(T-t)(|x|-R)+|x|^{2}} u\right\|_{L^{2}\left(Q_{R, T}\right)}+\left\|e^{\alpha(T-t)(|x|-R)+|x|^{2}} \nabla u\right\|_{L^{2}\left(Q_{R, T}\right)} \\
& \leqq\left\|e^{\alpha(T-t)(|x|-R)+|x|^{2}}\left(\Delta u+\partial_{t} u\right)\right\|_{L^{2}\left(Q_{R, T}\right)} \\
&+\left\|e^{|x|^{2}} \nabla u(., T)\right\|_{L^{2}\left(\mathbb{R}^{n} \backslash B_{R}\right)}
\end{aligned}
$$

hold for all $\alpha \geqq \alpha_{0}$ and $u \in C_{0}^{\infty}\left(Q_{R, T}\right)$ satisfying $u(., 0) \equiv 0$. 
The first Carleman inequality, based on ideas developed in [3-6], is used to prove that under the assumptions of Theorem 1 we have

$$
|u(x, t)|+|\nabla u(x, t)| \leqq N e^{-|x|^{2} /(N t)}
$$

for some $N>0$ in $Q_{6 R, T}$. This estimate enables us to apply the second Carleman inequality to the (slightly modified) function $u$, and obtain the result (for sufficiently small $T$ ) by letting $\alpha \rightarrow \infty$.Inequality (1.5) seems to be new.

As is usual in the context of $L^{2}$ Carleman estimates, we use suitable integration by parts to prove our main inequalities. The calculations can be organized either by using identities developed in [6], or by following more or less standard calculations with new dependent variables and commutators in the spirit of [8,9] or [21]. In this paper we will use the former method, which is based on Lemma 1 below. An alternative proof of Theorem 1 , which uses the latter method and a slightly modified version of (1.4) and (1.5) will appear elsewhere.

\section{Proof of the main result}

In what follows we assume that the functions entering the expressions below are "sufficiently regular" so that the quantities we consider are suitably well defined. For example, the assumption that the derivatives entering our formulae are distributional derivatives and are square integrable over bounded sets is sufficient.

Theorem 1. Assume that $u: Q_{R, T} \longrightarrow \mathbb{R}$ verifies in $Q_{R, T}$ the inequalities

$$
\left|\Delta u+\partial_{t} u\right| \leqq M(|u|+|\nabla u|) \quad \text { and } \quad|u(x, t)| \leqq M e^{M|x|^{2}}
$$

for some positive constants $M, T$ and $R$. Then, if $u(x, 0) \equiv 0$ in $\mathbb{R}^{n} \backslash B_{R}$, u vanishes identically in $Q_{R, T}$.

Though the proof of this result is given for real-valued functions, the arguments also work when $u$ is replaced by a vector-valued function $\omega: Q_{R, T} \longrightarrow \mathbb{R}^{n}$ verifying the same conditions and the inequality

$$
\left|\Delta \omega+\partial_{t} \omega\right| \leqq M(|\omega|+|\nabla \omega|) .
$$

This is because (2.1) is a triangular parabolic system with $\partial_{t}+\Delta$ as the principal part and the solutions $\omega$ to (2.1) verify the $L^{\infty}$ bounds [13] for real-valued subsolutions to parabolic equations: if $\omega \in W_{2, \mathrm{loc}}^{2,1}\left(Q_{R, T}\right)$ is a solution of (2.1), there is a constant $N$ depending on $M$ such that

$$
\sqrt{s}|\nabla \omega(y, s)|+|\omega(y, s)| \leqq \frac{N}{s^{\frac{n}{2}+1}} \int_{s}^{2 s} \int_{B_{\sqrt{s}}(y)}|\omega| d X
$$

when $|y|>2 \sqrt{s}+R$ and $0<s \leqq T / 2$.

To prove the result we need four lemmas. The Lemmas 1 through 3 are used to build the Carleman inequalities. In Lemma 4 it is shown that $u$ vanishes in $\mathbb{R}^{n} \backslash B_{R} \times[0, M]$ when $M$ is small, $R \geqq 1$ and $T=1$, and the general case follows using suitable parabolic rescalings and time translations.

Below $d X=d x d t$ and $\mathcal{I}$ denotes the identity matrix. 
Lemma 1. Assume that $u$ and $G$ are smooth functions on an open set in $\mathbb{R}^{n+1}$ and that $G$ is positive. Then, the following identity holds when $F=\left(\partial_{t} G-\Delta G\right) / G$ :

$$
\begin{aligned}
\nabla \cdot & {\left[2 \partial_{t} u G \nabla u+|\nabla u|^{2} \nabla G-2(\nabla G \cdot \nabla u) \nabla u+u F G \nabla u\right.} \\
& \left.+\frac{1}{2} u^{2} F \nabla G-\frac{1}{2} u^{2} G \nabla F\right]-\partial_{t}\left[|\nabla u|^{2} G+\frac{1}{2} u^{2} F G\right] \\
= & 2\left(\partial_{t} u-\nabla \log G \cdot \nabla u+\frac{1}{2} F u\right)\left(\Delta u+\partial_{t} u\right) G-2\left(\partial_{t} u-\nabla \log G \cdot \nabla u\right. \\
& \left.+\frac{1}{2} F u\right)^{2} G-\frac{1}{2} u^{2}\left(\partial_{t} F+\Delta F\right) G-2 D^{2}(\log G) \nabla u \cdot \nabla u G .
\end{aligned}
$$

Proof. The formula follows formally upon expanding the left-hand side with the product rule, multiplying out the products and squares on the right-hand side and comparing both outcomes.

The main ideas behind the previous argument come from the following observations. First,

$$
\begin{aligned}
2\left(\partial_{t} u-\nabla \log G \cdot \nabla u+\frac{1}{2} F u\right)\left(\Delta u+\partial_{t} u\right) G \\
\quad-2\left(\partial_{t} u-\nabla \log G \cdot \nabla u+\frac{1}{2} F u\right)^{2} G \\
=2\left(\partial_{t} u-\nabla \log G \cdot \nabla u+\frac{1}{2} F u\right)\left(\Delta u+\nabla \log G \cdot \nabla u-\frac{1}{2} F u\right) G,
\end{aligned}
$$

and the operators

$$
A=\partial_{t}-\nabla \log G \cdot \nabla+\frac{1}{2} F \quad \text { and } \quad S=\Delta+\nabla \log G \cdot \nabla-\frac{1}{2} F
$$

are respectively antisymmetric and symmetric in $L^{2}(G d X)$. We can now use the Rellich-Nečas identity with vector field $\nabla G$,

$$
2 \nabla G \cdot \nabla u \Delta u=\Delta G|\nabla u|^{2}-2 D^{2} G \nabla u \cdot \nabla u-\nabla \cdot\left(|\nabla u|^{2} \nabla G-2(\nabla G \cdot \nabla u) \nabla u\right),
$$

to compute the quadratic form associated with the commutator of these two operators in $L^{2}(G d X)$.

Now, integrating the formula in Lemma 1 over $\mathbb{R}^{n} \times[0, T]$ we get the following identity.

Lemma 2. Assume that $G$ is a smooth positive function in $Q_{R, T}, u \in C_{0}^{\infty}\left(Q_{R, T}\right)$ and set $F=\left(\partial_{t} G-\Delta G\right) / G$. Then, the following identity holds:

$$
\begin{aligned}
2 \int & \left(\partial_{t} u-\nabla \log G \cdot \nabla u+\frac{1}{2} F u\right)^{2} G d X+2 \int D^{2}(\log G) \nabla u \cdot \nabla u G d X \\
& +\frac{1}{2} \int u^{2}\left(\partial_{t} F+\Delta F\right) G d X \\
= & 2 \int\left(\Delta u+\partial_{t} u\right)\left(\partial_{t} u-\nabla \log G \cdot \nabla u+\frac{1}{2} F u\right) G d X \\
& +\left.\int|\nabla u|^{2} G d x\right|_{t=0} ^{t=T}+\left.\frac{1}{2} \int u^{2} F G d x\right|_{t=0} ^{t=T} .
\end{aligned}
$$


Thus, in general we can expect to control the $L^{2}(G d X)$ norm of $u$ and $\nabla u$ by the $L^{2}(G d X)$ norm of $\Delta u+\partial_{t} u$, where $\log G$ is convex and $\partial_{t} F+\Delta F>0$.

In particular, when $G=e^{2 \alpha(T-t)(|x|-R)+2|x|^{2}}$ the following inequalities hold on $Q_{R, T}$ for $\alpha>0$ sufficiently large depending on $n$ and $R$ :

$$
D^{2}(\log G) \geqq \mathcal{I}, \quad F \leqq 0 \quad \text { and } \quad \partial_{t} F+\Delta F \geqq 1 .
$$

Then, the second Carleman inequality (1.5) follows from Lemma 2, (2.2) and from the Cauchy-Schwarz inequality (which is used to handle the first integral on the right-hand side of the formula in Lemma 2).

The Carleman inequality (1.4) involves the function $G=e^{-|x|^{2} / 4 t}$. In this case, $D^{2}(\log G)=-\frac{1}{2 t} \mathcal{I}$ and $\log G$ is concave, but multiplying the identity in Lemma 1 by a suitable function depending on time, we can make the "commutator"of the two operators given below a positive operator. In particular, following the formula (2.3) below,

$$
\begin{aligned}
& \frac{\sigma^{1-\alpha}}{\dot{\sigma}}\left(\partial_{t} u-\nabla \log G \cdot \nabla u-\frac{\alpha \dot{\sigma}}{2 \sigma} u\right)\left(\Delta u+\partial_{t} u\right) G \\
& \quad-\frac{\sigma^{1-\alpha}}{\dot{\sigma}}\left(\partial_{t} u-\nabla \log G \cdot \nabla u-\frac{\alpha \dot{\sigma}}{2 \sigma} u\right)^{2} G \\
& =\frac{\sigma^{1-\alpha}}{\dot{\sigma}}\left(\partial_{t} u-\nabla \log G \cdot \nabla u-\frac{\alpha \dot{\sigma}}{2 \sigma} u\right)\left(\Delta u+\nabla \log G \cdot \nabla u+\frac{\alpha \dot{\sigma}}{2 \sigma} u\right) G,
\end{aligned}
$$

and the operators

$$
A=\partial_{t}-\nabla \log G \cdot \nabla-\frac{\alpha \dot{\sigma}}{2 \sigma} \quad \text { and } \quad S=\Delta+\nabla \log G \cdot \nabla+\frac{\alpha \dot{\sigma}}{2 \sigma}
$$

are respectively antisymmetric and symmetric in $L^{2}\left(\sigma^{-\alpha} G d X\right)$. Then, the quadratic form associated with the "commutator"with respect with the measure $\frac{\sigma^{1-\alpha}}{\dot{\sigma}}$ $G d X$ is calculated using the divergence theorem.

Finally, the parameter $a \in(0,1)$ in the Carleman inequality (1.4) is there to make rigorous the integration by parts done in its proof when $u \in C_{0}^{\infty}\left(\mathbb{R}^{n} \times[0,1)\right)$ and $u(x, 0) \equiv 0$ on $\mathbb{R}^{n}$. Setting there $a=0$ would only allow us to enter in (1.4) functions $u \in C_{0}^{\infty}\left(\mathbb{R}^{n} \times(0,1)\right)$ or vanishing to infinite order at $(y, 0)$ in the $(x, t)$-variable.

Lemma 3. Let $\alpha>0, G$ denote a positive caloric function in $\mathbb{R}^{n} \times[0,1]$ and $\sigma=\sigma(t)$ a positive non-decreasing function on $[0,1)$. Then, the following identity holds for all $u \in C_{0}^{\infty}\left(\mathbb{R}^{n} \times[0,1)\right)$ verifying $u(x, 0)=0$ on $\mathbb{R}^{n}$ :

$$
\begin{aligned}
& 2 \int \frac{\sigma^{1-\alpha}}{\dot{\sigma}}\left(\partial_{t} u-\nabla \log G \cdot \nabla u-\frac{\alpha \dot{\sigma}}{2 \sigma} u\right)^{2} G d X+\int \frac{\sigma^{1-\alpha}}{\dot{\sigma}} \mathcal{D}_{G} \nabla u \cdot \nabla u G d X \\
& =2 \int \frac{\sigma^{1-\alpha}}{\dot{\sigma}}\left(\Delta u+\partial_{t} u\right)\left(\partial_{t} u-\nabla \log G \cdot \nabla u-\frac{\alpha \dot{\sigma}}{2 \sigma} u\right) G d X,
\end{aligned}
$$

where $\mathcal{D}_{G}=\widehat{\log \frac{\sigma}{\dot{\sigma}}} \mathcal{I}+2 D^{2}(\log G)$. 
Proof. Replacing in Lemma 1 the function $G$ by $\sigma^{-\alpha} G$, we have $F=-\alpha \widehat{\log \sigma}$ and the identity

$$
\begin{aligned}
\sigma^{-\alpha} \nabla \cdot\left[2 \partial_{t} u G \nabla u+|\nabla u|^{2} \nabla G-2(\nabla G \cdot \nabla u) \nabla u-\frac{\alpha \dot{\sigma}}{\sigma} u G \nabla u-\frac{\alpha \dot{\sigma}}{2 \sigma} u^{2} \nabla G\right] \\
=2 \sigma^{-\alpha}\left(\partial_{t} u-\nabla \log G \cdot \nabla u-\frac{\alpha \dot{\sigma}}{2 \sigma} u\right)\left(\Delta u+\partial_{t} u\right) G \\
-2 \sigma^{-\alpha}\left(\partial_{t} u-\nabla \log G \cdot \nabla u-\frac{\alpha \dot{\sigma}}{2 \sigma} u\right)^{2} G+\frac{\alpha}{2} \sigma^{-\alpha} \frac{\ddot{\log \sigma}}{2} u^{2} G \\
-2 \sigma^{-\alpha} D^{2}(\log G) \nabla u \cdot \nabla u G+\partial_{t}\left[\sigma^{-\alpha}|\nabla u|^{2} G-\frac{\alpha \dot{\sigma}}{2 \sigma} \sigma^{-\alpha} u^{2} G\right] .
\end{aligned}
$$

Multiplying (2.3) by $\frac{\sigma}{\dot{\sigma}}$ and using the identities

$$
\begin{aligned}
\left(\frac{\sigma}{\dot{\sigma}}\right) \widehat{\ddot{\log \sigma}}= & -\widehat{\log \frac{\sigma}{\dot{\sigma}}}, \frac{\sigma}{\dot{\sigma}} \partial_{t}\left[\sigma^{-\alpha}|\nabla u|^{2} G-\frac{\alpha \dot{\sigma}}{2 \sigma} \sigma^{-\alpha} u^{2} G\right] \\
= & \partial_{t}\left[\frac{\sigma^{1-\alpha}}{\dot{\sigma}}|\nabla u|^{2} G-\frac{\alpha \sigma^{-\alpha}}{2} u^{2} G\right]-\frac{\sigma^{1-\alpha}}{\dot{\sigma}} \log \frac{\dot{\sigma}}{\dot{\sigma}}|\nabla u|^{2} G \\
& +\frac{\alpha \sigma^{-\alpha}}{2} \hat{\log \frac{\sigma}{\dot{\sigma}}} u^{2} G,
\end{aligned}
$$

it follows that

$$
\begin{aligned}
& \frac{\sigma^{1-\alpha}}{\dot{\sigma}} \nabla \cdot\left[2 \partial_{t} u G \nabla u+|\nabla u|^{2} \nabla G-2(\nabla G \cdot \nabla u) \nabla u-\frac{\alpha \dot{\sigma}}{\sigma} u G \nabla u-\frac{\alpha \dot{\sigma}}{2 \sigma} u^{2} \nabla G\right] \\
& =\frac{2 \sigma^{1-\alpha}}{\dot{\sigma}}\left(\partial_{t} u-\nabla \log G \cdot \nabla u-\frac{\alpha \dot{\sigma}}{2 \sigma} u\right)\left(\Delta u+\partial_{t} u\right) G \\
& \quad-\frac{2 \sigma^{1-\alpha}}{\dot{\sigma}}\left(\partial_{t} u-\nabla \log G \cdot \nabla u-\frac{\alpha \dot{\sigma}}{2 \sigma} u\right)^{2} G-\frac{\sigma^{1-\alpha}}{\dot{\sigma}} \mathcal{D}_{G} \nabla u \cdot \nabla u G \\
& \quad+\partial_{t}\left[\frac{\sigma^{1-\alpha}}{\dot{\sigma}}|\nabla u|^{2} G-\frac{\alpha \sigma^{-\alpha}}{2} u^{2} G\right],
\end{aligned}
$$

and the identity in Lemma 2 follows upon integrating (2.4) over $\mathbb{R}^{n} \times[0,1]$.

When $a \in(0,1)$ and taking respectively in Lemma 3 as $G$ and $\sigma$ the functions $G_{a}=(t+a)^{-n / 2} e^{-|x|^{2} / 4(t+a)}$ and $\sigma_{a}=(t+a) e^{-(t+a) / 3}$, we have

$$
\frac{1}{3 e}(t+a) \leqq \sigma_{a}(t) \leqq t+a, \quad \frac{1}{3 e} \leqq \dot{\sigma}_{a}(t) \leqq 1 \quad \text { and } \quad \mathcal{D}_{G_{a}} \geqq \frac{1}{3} \mathcal{I}
$$

when $t \in(0,1)$ and $(x, t) \in \mathbb{R}^{n} \times(0,1)$. With these choices, the integrations by parts done in the derivation of Lemma 3 are valid when $u \in C_{0}^{\infty}\left(\mathbb{R}^{n} \times[0,1)\right)$ and $u(x, 0) \equiv 0$ on $\mathbb{R}^{n}$, and from Lemma $3,(2.5)$ and the Cauchy-Schwarz inequality, 


$$
\left\|\sigma_{a}^{-\alpha} G_{a}^{1 / 2} \nabla u\right\|_{L^{2}\left(\mathbb{R}^{n} \times(0,1)\right)} \lesssim\left\|\sigma_{a}^{-\alpha} G_{a}^{1 / 2}\left(\Delta u+\partial_{t} u\right)\right\|_{L^{2}\left(\mathbb{R}^{n} \times(0,1)\right)} .
$$

Multiplying the identity

$$
\left(\Delta+\partial_{t}\right)\left(u^{2}\right)=2 u\left(\Delta u+\partial_{t} u\right)+2|\nabla u|^{2}
$$

by $\sigma_{a}^{-2 \alpha} G_{a}$, then integrating by parts the operator $\Delta+\partial_{t}$ acting on $u^{2}$ over the other terms in the corresponding integral over $\mathbb{R}^{n} \times(0,1)$, and using the Cauchy-Schwarz inequality to handle the cross term, (2.5) and (2.6), we find that the following inequality holds when $\alpha>0$ :

$$
\begin{aligned}
& \sqrt{\alpha}\left\|\sigma_{a}^{-\alpha-1 / 2} G_{a}^{1 / 2} u\right\|_{L^{2}\left(\mathbb{R}^{n} \times(0,1)\right)}+\left\|\sigma_{a}^{-\alpha} G_{a}^{1 / 2} \nabla u\right\|_{L^{2}\left(\mathbb{R}^{n} \times(0,1)\right)} \\
& \quad \lesssim\left\|\sigma_{a}^{-\alpha} G_{a}^{1 / 2}\left(\Delta u+\partial_{t} u\right)\right\|_{L^{2}\left(\mathbb{R}^{n} \times(0,1)\right)} .
\end{aligned}
$$

This proves the Carleman inequality (1.4) upon replacing $\alpha$ by $\alpha-\frac{n}{4}$ and observing that the previous inequality is invariant under translations of the space variable.

Lemma 4. Assume that $u$ satisfies in $\mathbb{R}^{n} \backslash B_{R} \times[0,1]$

$$
\left|\Delta u+\partial_{t} u\right| \leqq \varepsilon(|u|+|\nabla u|), \quad|u(x, t)| \leqq e^{\varepsilon|x|^{2}},
$$

and $u(x, 0) \equiv 0$ in $\mathbb{R}^{n} \backslash B_{R}$ for some $R \geqq 1$. Then, there is $\varepsilon(n)>0$ such that $u$ is identically zero in $\mathbb{R}^{n} \backslash B_{R} \times[0, \varepsilon]$ when $\varepsilon \leqq \varepsilon(n)$.

Proof. In order to obtain this result we first show that there exists $\varepsilon(n)>0$ and a constant $N=N(n)$ such that any such function verifies, in $\mathbb{R}^{n} \backslash B_{6 R} \times\left[0, \frac{1}{N}\right]$,

$$
|u(y, s)|+|\nabla u(y, s)| \leqq N e^{-|y|^{2} /(N s)}\left(1+\|u\|_{L^{\infty}\left(B_{4 R} \backslash B_{R} \times[0,1]\right)}\right)
$$

when $\varepsilon \leqq \varepsilon(n)$.

Assuming that $u$ verifies (2.7), $|y| \geqq 6 R, a \in(0,1)$ and that $r \geqq 4|y|$ is a large number, we will apply the first Carleman inequality (1.4) to $u_{r}(x, t)=$ $u(x, t) \varphi(t) \psi_{r}(x)$, where $\varphi \in C^{\infty}(\mathbb{R})$ and $\psi_{r} \in C_{0}^{\infty}\left(\mathbb{R}^{n}\right)$ satisfy $\varphi=1$ for $t \leqq 1 / 2$, $\varphi=0$ for $t \geqq 3 / 4, \psi_{r}=1$ for $3 R \leqq|x| \leqq 2 r, \psi_{r}=0$ for $|x| \leqq 2 R$ and $|x| \geqq 3 r$, $0 \leqq \varphi, \psi \leqq 1$, and taking $\alpha=k, k \in \mathbb{N}$, in (1.4). With these definitions we have

$$
\begin{aligned}
& \left\|\sigma_{a}^{-k-1 / 2} e^{-|x-y|^{2} / 8(t+a)} u_{r}\right\|_{2}+\left\|\sigma_{a}^{-k} e^{-|x-y|^{2} / 8(t+a)} \nabla u_{r}\right\|_{2} \\
& \quad \lesssim\left\|\sigma_{a}^{-k} e^{-|x-y|^{2} / 8(t+a)}\left(\Delta+\partial_{t}\right)\left(u_{r}\right)\right\|_{2} .
\end{aligned}
$$

On the other hand,

$$
\begin{aligned}
& \left|\left(\Delta+\partial_{t}\right)\left(u_{r}\right)\right| \leqq \varepsilon\left(\left|u_{r}\right|+\left|\nabla u_{r}\right|\right)+\left|\varphi^{\prime} u\right| \\
& \quad+\varphi\left[|u|\left(\left|\Delta \psi_{r}\right|+\left|\nabla \psi_{r}\right|\right)+2\left|\nabla \psi_{r}\right||\nabla u|\right],
\end{aligned}
$$


and from (2.9) it is possible to hide the first term on the right-hand side of (2.10) in the left-hand side of (2.9) when $\varepsilon$ is sufficiently small, and obtain, for $k \geqq 0$,

$$
\begin{aligned}
& \left\|\sigma_{a}^{-k-1 / 2} e^{-|x-y|^{2} / 8(t+a)} u_{r}\right\|_{2}+\left\|\sigma_{a}^{-k} e^{-|x-y|^{2} / 8(t+a)} \nabla u_{r}\right\|_{2} \\
& \lesssim\left\|\sigma_{a}^{-k} e^{-|x-y|^{2} / 8(t+a)} u\right\|_{L^{2}\left(\mathbb{R}^{n} \backslash B_{R} \times\left[\frac{1}{2}, \frac{3}{4}\right]\right)} \\
& \quad+\left\|\sigma_{a}^{-k} e^{-|x-y|^{2} / 8(t+a)}(|u|+|\nabla u|)\right\|_{L^{2}\left(B_{3 R} \backslash B_{2 R} \times\left[0, \frac{3}{4}\right]\right)} \\
& \quad+\left\|\sigma_{a}^{-k} e^{-|x-y|^{2} / 8(t+a)}(|u|+|\nabla u|)\right\|_{L^{2}\left(B_{3 r} \backslash B_{2 r} \times\left[0, \frac{3}{4}\right]\right)} .
\end{aligned}
$$

The standard $L^{\infty}$ bounds for the gradient of subsolutions to parabolic equations [13] imply that there is a constant depending on $n$ and $k$ such that

$$
\begin{aligned}
& \left\|\sigma_{a}^{-k} e^{-|x-y|^{2} / 8(t+a)}(|u|+|\nabla u|)\right\|_{L^{2}\left(B_{3 r} \backslash B_{2 r} \times\left[0, \frac{3}{4}\right]\right)} \\
& \quad \lesssim a^{-k} e^{-r^{2}}\|u\|_{L^{\infty}\left(\left(B_{4 r} \backslash B_{r}\right) \times[0,1]\right)},
\end{aligned}
$$

and from the growth condition (2.7), the right-hand side of this inequality tends to zero as $r \rightarrow+\infty$ when $\varepsilon<1 / 16$. Then, letting first $r \rightarrow+\infty$ and then $a \rightarrow 0$ in (2.11), it follows from (2.5) that there is a universal constant $N=N(n)$ such that, for $k \geqq 0$,

$$
\begin{aligned}
&\left\|t^{-k-1 / 2} e^{-|x-y|^{2} / 8 t} u\right\|_{L^{2}\left(\mathbb{R}^{n} \backslash B_{2 R} \times\left[0, \frac{1}{2}\right]\right)}+\left\|t^{-k} e^{-|x-y|^{2} / 8 t} \nabla u\right\|_{L^{2}\left(\mathbb{R}^{n} \backslash B_{2 R} \times\left[0, \frac{1}{2}\right]\right)} \\
& \leqq N^{k}\left\|e^{-|x-y|^{2} / 8 t} u\right\|_{L^{2}\left(\mathbb{R}^{n} \backslash B_{R} \times\left[\frac{1}{2}, \frac{3}{4}\right]\right)} \\
&+N^{k}\left\|t^{-k} e^{-|x-y|^{2} / 8 t}(|u|+|\nabla u|)\right\|_{L^{2}\left(B_{3 R} \backslash B_{2 R} \times\left[0, \frac{3}{4}\right]\right)}
\end{aligned}
$$

From (2.7),

$$
\left\|e^{-|x-y|^{2} / 8 t} u\right\|_{L^{2}\left(\mathbb{R}^{n} \backslash B_{R} \times\left[\frac{1}{2}, \frac{3}{4}\right]\right)} \lesssim\left\|e^{\varepsilon|x|^{2}-\frac{|x-y|^{2}}{6}}\right\|_{L^{2}\left(\mathbb{R}^{n}\right)} \lesssim e^{|y|^{2}} .
$$

On the other hand, $e^{-|x-y|^{2} / 8 t} \leqq e^{-|y|^{2} / 16 t}$ when $x \in B_{3 R} \backslash B_{2 R}$ and from Stirling's formula [1]

$$
\max _{t>0} t^{-k} e^{-|y|^{2} / 16 t}=|y|^{-2 k}(16 k)^{k} e^{-k} \leqq|y|^{-2 k} N^{k} k !,
$$

and from this fact and the standard $L^{\infty}$ bounds for the gradient of subsolutions to parabolic inequalities [13] we have

$$
\begin{aligned}
& \left\|t^{-k} e^{-|x-y|^{2} / 8 t}(|u|+|\nabla u|)\right\|_{L^{2}\left(B_{3 R} \backslash B_{2 R} \times\left[0, \frac{3}{4}\right]\right)} \\
& \quad \leqq N^{k} k !|y|^{-2 k}\|u\|_{L^{\infty}\left(B_{4 R} \backslash B_{R} \times[0,1]\right)} .
\end{aligned}
$$

Then, (2.12)-(2.14) and (2.7) imply that there is a constant $N=N(n)$ such that, for $|y| \geqq 6 R$ and $k \geqq 0$,

$$
\left\|t^{-k} e^{-|x-y|^{2} / 8 t} u\right\|_{L^{2}\left(\left(\mathbb{R}^{n} \backslash B_{2 R} \times\left[0, \frac{1}{2}\right]\right)\right.} \leqq N^{k}\left(k !|y|^{-2 k}\|u\|_{L^{\infty}\left(B_{4 R} \backslash B_{R} \times[0,1]\right)}+e^{|y|^{2}}\right),
$$


and multiplying this inequality by $|y|^{2 k} /\left((2 N)^{k} k !\right)$ and adding up in $k \geqq 0$,

$$
\left\|e^{|y|^{2} /(4 N t)} e^{-|x-y|^{2} / 8(t+a)} u\right\|_{L^{2}\left(\left(\mathbb{R}^{n} \backslash B_{2 R} \times\left[0, \frac{1}{8 N}\right]\right)\right.} \lesssim 1+\|u\|_{L^{\infty}\left(B_{4 R} \backslash B_{R} \times[0,1]\right)} .
$$

Then, from standard estimates for subsolutions of parabolic inequalities [13],

$$
|u(y, s)|+\sqrt{s}|\nabla u(y, s)| \lesssim \frac{1}{s^{\frac{n}{2}+1}} \int_{s}^{2 s} \int_{B_{\sqrt{s}}(y)}|u| d X
$$

and the claim (2.8) follows from (2.15) and (2.16).

Now, we apply the second Carleman inequality (1.5) to $u_{a, r}=u \psi_{a, r}$, where $0<a<1, r>0$ is a large number, $\psi_{a, r} \in C_{0}^{\infty}\left(\mathbb{R}^{n}\right)$ verifies $\psi_{a, r}=0$ for $|x| \leqq(1+a) R$ or $|x| \geqq 2 r$ and $\psi_{a, r}=1$ for $(1+2 a) R \leqq|x| \leqq r$, and choosing $T=4 \varepsilon$, where $0<\varepsilon \leqq 1 /(10 N)$ and $N$ is the constant in (2.8). In particular, we have

$$
\begin{aligned}
& \left\|e^{\alpha(4 \varepsilon-t)(|x|-R)+|x|^{2}} u_{a, r}\right\|_{L^{2}\left(Q_{R, 4 \varepsilon}\right)}+\left\|e^{\alpha(4 \varepsilon-t)(|x|-R)+|x|^{2}} \nabla u_{a, r}\right\|_{L^{2}\left(Q_{R, 4 \varepsilon}\right)} \\
& \leqq \\
& \quad\left\|e^{\alpha(4 \varepsilon-t)(|x|-R)+|x|^{2}}\left(\Delta+\partial_{t}\right)\left(u_{a, r}\right)\right\|_{L^{2}\left(Q_{R, 4 \varepsilon}\right)} \\
& \quad+\left\|e^{|x|^{2}} \nabla u_{a, r}(., 4 \varepsilon)\right\|_{L^{2}\left(\mathbb{R}^{n}\right)}
\end{aligned}
$$

As before,

$$
\begin{aligned}
\left|\left(\Delta+\partial_{t}\right)\left(u_{a, r}\right)\right| \leqq & \varepsilon\left(\left|u_{a, r}\right|+\left|\nabla u_{a, r}\right|\right) \\
& +|u|\left(\left|\Delta \psi_{a, r}\right|+\left|\nabla \psi_{a, r}\right|\right)+2\left|\nabla \psi_{a, r}\right||\nabla u|,
\end{aligned}
$$

and the first term on the right-hand side of (2.18) can be hidden in the left-hand side of (2.17) when $\varepsilon$ is sufficiently small. These imply that the following inequality holds with a constant depending on $a>0$ :

$$
\begin{aligned}
e^{10 \alpha \varepsilon a R}\|u\|_{L^{2}\left(B_{r} \backslash B_{(1+10 a) R} \times[0, \varepsilon]\right) \lesssim} & e^{8 \alpha \varepsilon r+4 r^{2}}\||u|+|\nabla u|\|_{L^{2}\left(B_{2 r} \backslash B_{r} \times[0,4 \varepsilon]\right)} \\
& +e^{8 \alpha \varepsilon a R}\||u|+|\nabla u|\|_{L^{2}\left(B_{(1+2 a) R} \backslash B_{(1+a) R} \times[0,4 \varepsilon]\right)} \\
& +\left\|e^{|x|^{2}} u(., 4 \varepsilon)\right\|_{L^{2}\left(B_{2 r} \backslash B_{(1+a) R}\right)} \\
& +\left\|e^{|x|^{2}} \nabla u(., 4 \varepsilon) \mid\right\|_{L^{2}\left(B_{2 r} \backslash B_{(1+a) R}\right)} .
\end{aligned}
$$

This inequality and (2.8) imply that for some constant depending on $a$ and $\varepsilon$,

$$
\|u\|_{L^{2}\left(B_{r} \backslash B_{(1+10 a) R} \times[0, \varepsilon]\right)} \lesssim e^{\alpha r-r^{2}}+e^{-2 \alpha \varepsilon a R} .
$$

Then, letting first $r \rightarrow+\infty$, then $\alpha \rightarrow+\infty$ and finally $a \rightarrow 0^{+}$in the last inequality, gives $u \equiv 0$ in $\mathbb{R}^{n} \backslash B_{R} \times[0, \varepsilon]$. 
In general, when $u$ satisfies the assumptions in Theorem 1 , there is $\delta>0$ such that the function $u\left(\delta x, \delta^{2} t\right) / M$, also denoted $u$, verifies (2.7) in $\mathbb{R}^{n} \backslash B_{R} \times[0, T]$ for some new $R \geqq 1$ and $T \geqq 1$ and with $\varepsilon$ as small as we like. If $T \geqq 1$, Lemma 4 gives $u \equiv 0$ in $\mathbb{R}^{n} \backslash B_{R} \times[0, \varepsilon]$. Then, $u(x, t+\varepsilon)$ satisfies (2.7) in $\mathbb{R}^{n} \backslash B_{R} \times[0, T-\varepsilon]$, and if $T-\varepsilon \geqq 1$ we get $u \equiv 0$ in $\mathbb{R}^{n} \backslash B_{R} \times[0,2 \varepsilon]$. Proceeding in this way, we find $a \geqq 0$ such that $u \equiv 0$ in $\mathbb{R}^{n} \backslash B_{R} \times[0, a]$ and $0<T-a \leqq 1$. Setting $a_{0}=a$ and $u_{k}(x, t)=u\left(\sqrt{T-a_{k}} x,\left(T-a_{k}\right) t+a_{k}\right)$ when $k \geqq 0, u_{k}$ verifies (2.7), and Lemma 4 implies that $u \equiv 0$ in $\mathbb{R}^{n} \backslash B_{R} \times\left[0, a_{k+1}\right]$, where $a_{k+1}=(1-\varepsilon) a_{k}+T \varepsilon$. The sequence $\left\{a_{k}\right\}$ is non-decreasing and $0<a_{k}<T$ for all $k \geqq 0$. These two facts imply that $\lim _{k \rightarrow+\infty} a_{k}=T$, and prove the theorem.

Acknowledgements. The research of L. ESCAURIAZA was supported by Spanish Government grant BFM 2001-0458 and by the European Commission via the network Harmonic Analysis and Related Problems, project number RTN2-2001-00315. G. SEREGIN wishes to acknowledge the hospitality of the School of Mathematics at the University of Minnesota, where a part of this work was carried out. The research of V. ŠVERÁK was supported in part by grant DMS 9877055 from the NSF.

\section{References}

1. AhLFORS, L.V.: Complex Analysis. McGraw-Hill, 1966

2. CHEN, X.Y.: A strong unique continuation theorem for parabolic equations. Math. Ann. 311, 603-630 (1996)

3. EsCAURIAZA, L.: Carleman inequalities and the heat operator. Duke Math. J. 104, 113127 (2000)

4. EscauriazA, L., VegA, L.: Carleman inequalities and the heat operator II. Indiana U. Math. J. 50, 1149-1169 (2001)

5. EsCAuriaZA, L., FernáNDEZ, F.J.: Unique continuation for parabolic operators. (to appear)

6. FERNÁNDEZ, F.J.: Unique continuation for parabolic operators II. (to appear)

7. Hopf, E.: Über die Anfangswertaufgabe für die hydrodynamischen Grundgleichungen. Math. Nachr. 4, 213-231 (1950)

8. HÖRMANDER, L.: Linear Partial Differential Operators. Springer, 1963

9. HÖRMANDER, L.: Uniqueness theorems for second order elliptic differential equations. Communications in PDE 8, 21-64 (1983)

10. JONES, B.F.: A fundamental solution of the heat equation which is supported in a strip. J. Math. Anal. Appl. 60, 314-324 (1977)

11. KATO, T.: Strong $L^{p}$-solutions of the Navier-Stokes equations in $\mathbb{R}^{m}$ with applications to weak solutions. Math. Zeit. 187, 471-480 (1984)

12. LADYZHENSKAYA, O.A.: Mathematical problems of the dynamics of viscous incompressible fluids. Gordon and Breach, 1969

13. LADYŽEnSKAJA, O.A., SOlONNIKOV, V.A., URAlCEVA, N.N.: Linear and quasilinear equations of parabolic type. Translations of Mathematical Monographs, Amer. Math. Soc., 1968

14. Lin, F.H.: A uniqueness theorem for parabolic equations. Comm. Pure Appl. Math. 42, 125-136 (1988)

15. LiTTMAN, W.: Boundary control theory for hyperbolic and parabolic partial differential equations with constant coefficients. Annali Scuola Norm. Sup. Pisa Serie IV 3, 567-580 (1978)

16. Micu, S., ZUAZUA, E.: On the lack of null-controllability of the heat equation on the half space. Portugaliae Mathematica 58, 1-24 (2001) 
17. Poon, C.C.: Unique continuation for parabolic equations. Comm. Partial Differential Equations 21, 521-539 (1996)

18. SAUT, J.C., SCHEURER, E.: Unique continuation for evolution equations. J. Differential Equations 66, 118-137 (1987)

19. SEREGIN, G., ŠVERÁK, V.: The Navier-Stokes equations and backward uniqueness. (to appear)

20. SoGGe, C.D.: A unique continuation theorem for second order parabolic differential operators. Ark. Mat. 28, 159-182 (1990)

21. TREVES, F.: Linear Partial Differential Equations. Gordon and Breach, 1970

\author{
Dpto. de Matemáticas \\ UPV/EHU \\ 48080 Bilbao, Spain \\ e-mail: mtpeszul@lg.ehu.es \\ and \\ Steklov Institute of Mathematics \\ St.Peterburg, Russia \\ e-mail: seregin@pdmi.ras.ru \\ and \\ School of Mathematics \\ University of Minnesota \\ Minneapolis, USA \\ e-mail:sverak@math.umn.edu
}

(Accepted February 7, 2003)

Published online May 28, 2003 - (C) Springer-Verlag (2003) 